\title{
Optimal Control of Agricultural Insects with a Stage-Structured Model
}

\author{
Baolin Kang, ${ }^{1,2}$ Mingfeng He, ${ }^{1}$ and Bing Liü \\ ${ }^{1}$ School of Mathematical Sciences, Dalian University of Technology, Dalian, Liaoning 116029, China \\ ${ }^{2}$ Department of Mathematics, Anshan Normal University, Anshan, Liaoning 114007, China
}

Correspondence should be addressed to Baolin Kang; shangjin1@126.com

Received 13 May 2013; Accepted 8 July 2013

Academic Editor: Hai-Feng Huo

Copyright (c) 2013 Baolin Kang et al. This is an open access article distributed under the Creative Commons Attribution License, which permits unrestricted use, distribution, and reproduction in any medium, provided the original work is properly cited.

\begin{abstract}
A stage-structured pest control model with impulse effects by state feedback control is formulated, and a semicontinuous dynamic system and its successor functions are defined. The sufficient conditions of existence and attractiveness of order one periodic solution are obtained by the method of successor functions. The superiority of the state feedback control strategy in this paper is that we only need to monitor the sum of immature and mature pest populations. Moreover, our results show that our method used in this paper is more efficient and easier than the existing methods.
\end{abstract}

\section{Introduction}

Insects are among the most diverse group of animals on the planet and include more than a million described species and represent more than half of all known living organisms. The number of extant species is estimated to be at between six and ten million and potentially represents over 90 percent of the differing metazoan life forms on Earth. They may be found in nearly all environments, although only a small number of species occur in the oceans, a habitat dominated by another arthropod group, the crustaceans. Some insects damage crops by feeding on sap, leaves, or fruits, a few bite humans and livestock, alive and dead, to feed on blood and some are capable of transmitting diseases to humans, pets and livestock. So, humans regard certain insects as pests, and attempt to control them using a host of techniques. Pest control is very important because out of control pests can wreak havoc. For example, many countries in the world suffer deeply from plagues of locusts each year.

In pest management, we can control the pest population by many methods, such as spraying pesticides and releasing natural enemies. Among the methods, chemical pest control is still the predominant type of pest control today, although its long-term effects led to a renewed interest in traditional and biological pest controls towards the end of the 20th century.
For most pests, such as aphid and silkworm, the survival rate has a relation with their age and development stage. In recent years, various stage-structure models have been proposed and studied for populations [1-12]. We suppose a stage-structure model as follows:

$$
\begin{aligned}
& \frac{d x}{d t}=-a x+b y, \\
& \frac{d y}{d t}=c x-d y,
\end{aligned}
$$

where $x(t)$ and $y(t)$ represent the densities of immature and mature pest populations, respectively; $a, b, c$, and $d$ are positive constants; $a$ denotes the sum of the rate of an immature population turning into a mature population and the death rate of the immature pest population; $b$ and $d$ denote the birth rate of the immature pest population and the death rate of the mature pest population, respectively; $c$ denotes the rate of an immature population turning into a mature population. The dynamics property of system (1) is very simple. The unique equilibrium point of system (1) is $O(0,0)$. In the case of $a d-$ $b c>0, O(0,0)$ is stable, so no pest control measure is needed. But in the case of $a d-b c<0, O(0,0)$ is a saddle with a separatrix $\Gamma: k_{1} x$, where $k_{1}=\left((a-d)+\sqrt{(a-d)^{2}+4 b c}\right) / 2 b$. 
So the effective measures are necessary to control the pest population.

Apparently, when the pesticides are sprayed, it is natural to assume that both the mature and immature pest populations diminish abruptly; that is, impulse occurs, and we can use an impulsive differential equation to model the process of spraying pesticides. The theory of impulsive differential systems has been developed by numerous mathematicians [13-15]. There are three kinds of typical impulsive differential equations [13]: (1) systems with impulses at fixed time; (2) systems with impulses at variable time; (3) autonomous systems with impulses. In recent years, most of researches on IDEs concern systems of types 1 [16-23]. However, the drawbacks of type (1) lie in neglecting growth law of the pest and the cost of management. We must control the amount of pesticide and use them in the crucial phase in order to maximize the benefits of pesticides. Therefore we should do the insect survey well, establish the reasonable control index, reduce the times of spraying, and improve the economic and ecological benefits. Today, some researchers also propose the way of the state feedback strategy by IDEs systems of types 3 , such as in [21] and the economic threshold (ET) is mentioned and defined in [22]. However, the shortcoming of state feedback control strategy in [21], is the that pesticides might be applied only when the density of the mature pests population reaches ET, which can lead to the sum of pests beyond the ET because of the overlook of immature pests. Considering the previous factors, we formulate the model as follows:

$$
\begin{aligned}
& \frac{d x}{d t}=-a x+b y, \quad x+y \neq h, \\
& \frac{d y}{d t}=c x-d y, \\
& \begin{array}{l}
\Delta x=-\alpha x, \\
\Delta y=-\beta y, x+y=h,
\end{array}
\end{aligned}
$$

where $a, b, c, d, h>0 . \Delta x=x\left(t^{+}\right)-x(t)$ and $\Delta y=y\left(t^{+}\right)-y(t)$; $\alpha$ and $\beta \in(0,1)$ represent the proportion of killed immature and mature pests by spraying pesticides, respectively; $h$ represents the ET of the pest totality. System (2) has been studied by Jiang et al. [23], and they obtained the sufficient conditions of existence and stability of periodic solutions by means of the sequence convergence rule and the analogue of the Poincare criterion. In this paper, we improve the demonstration method of [23] via the successor function.

The organization of this paper is as follows. In the next section, we give some important definitions and auxiliary lemmas. In Section 3, the existence of one order periodic solution is obtained by using successor function. In Section 4, we discuss the attractiveness of order one periodic solution under its existence conditions by means of sequence convergence rules and qualitative analysis. Finally, by numerical simulation, we can confirm our theoretical conclusions and give a brief discussion.

\section{Definitions and Lemmas}

Definition 1. A triple $\left(X, \Pi, R_{+}\right)$is said to be a semidynamical system if $X$ is a metric space, $R_{+}$is the set of all nonnegative real, and $\Pi(P, t): X \times R_{+} \rightarrow X$ is a continuous map such that:

(i) $\Pi(P, 0)=P$ for all $P \in X$;

(ii) $\Pi(P, t)$ is continuous for $t$ and $s$;

(iii) $\Pi(\Pi(P, t), s)=\Pi(P, t+s)$ for all $P \in X$ and $t, s \in R_{+}$.

Sometimes a semidynamical system $\left(X, \Pi, R_{+}\right)$is denoted by $(X, \Pi)$.

Definition 2. Assume that

(i) $(X, \Pi)$ is a semidynamical system;

(ii) $M$ is a nonempty subset of $X$;

(iii) function $I: M \rightarrow X$ is continuous and for any $P \in$ $M$, and there exists a $\varepsilon>0$ such that for any $0<|t|<$ $\varepsilon, \Pi(P, t) \notin M$.

Then, $(X, \Pi, M, I)$ is called an impulsive semidynamical system.

For any $P \in X$, the function $\Pi_{P}: R_{+} \rightarrow X$ defined as $\Pi_{P}(t)=\Pi(P, t)$ is continuous, and we call $\Pi_{P}(t)$ the trajectory passing through point $P$. The set $C^{+}(P)=\{\Pi(P, t) \mid 0 \leq$ $t<+\infty\}$ is called positive semitrajectory of point $P$, for any subset $M$ of $X$; we define $M^{+}(P)=C^{+}(P) \cap M-\{P\}$. The set $C^{-}(P)=\{\Pi(P, t) \mid-\infty<t \leq 0\}$ is called the negative semitrajectory of point $P$.

Let $\Pi(P, t)$ be the trajectory of differential dynamical system $d x / d t=f(x)$ passing through the point $P$. Now we give the trajectory $\widetilde{\Pi}(P, t)$ of the following impulsive semidynamical system:

$$
\begin{aligned}
& \frac{d x}{d t}=f(x), \quad x \notin M, \\
& x^{+}=I(x), \quad x \in M .
\end{aligned}
$$

Definition 3. Let $(X, \Pi, M, I)$ be an impulsive semidynamical system corresponding to the system (3), and, for any $P \in$ $X-M$, the trajectory $\widetilde{\Pi}(P, t):(X-M) \times R_{+} \rightarrow X$ passing through point $P$ is defined as follows:

$$
\text { if } M^{+}(p)=\emptyset, \widetilde{\Pi}(P, t)=\Pi(P, t) \text {, for } t \in R_{+} \text {; }
$$

if $M^{+}(p) \neq \emptyset$, there exists a $P_{1} \in M^{+}(P)$, and $T_{0} \in R_{+}$ such that $\Pi\left(P, T_{0}\right)=P_{1} \in M$ and $0<t<T_{0}, \Pi(P, t) \notin$ $M$. Let $I\left(P_{1}\right)=I\left(\Pi\left(P, T_{0}\right)\right)=P_{1}^{+} \in N$; then for $0 \leq$ $t \leq T_{0}$,

$$
\widetilde{\Pi}(P, t)= \begin{cases}\Pi(P, t), & 0 \leq t<T_{0}, \\ P_{1}^{+}, & t=T_{0},\end{cases}
$$

if $M^{+}\left(P_{1}\right)=\emptyset, \widetilde{\Pi}\left(P_{1}^{+}, t\right)=\Pi\left(P_{1}^{+}, t-T_{0}\right), t \geq T_{0}$;

if $M^{+}\left(P_{1}^{+}\right) \neq \emptyset$, there exists a $P_{2} \in M^{+}\left(P_{1}^{+}\right)$, and $T_{1} \in$ $R_{+}$such that $\Pi\left(P_{1}^{+}, T_{1}\right)=P_{2} \in M$ and $T_{0}<t<T_{0}+T_{1}$, $\Pi\left(P_{1}^{+}, t\right) \notin M$. Let $I\left(P_{2}\right)=I\left(\Pi\left(P_{1}^{+}\right), T_{1}\right)=P_{2}^{+} \in N$; then for $T_{0} \leq t \leq T_{0}+T_{1}$,

$\widetilde{\Pi}(P, t)= \begin{cases}\Pi\left(P_{1}^{+}, t-T_{0}\right), & T_{0} \leq t<T_{0}+T_{1}, \\ P_{2}^{+}, & t=T_{0}+T_{1} .\end{cases}$ 
Repeat the above process; then there exists an $N_{0}$ such that $M^{+}\left(P_{N_{0}}^{+}\right)=\emptyset$ or for any $n \in Z_{+}=\{1,2, \ldots\},, M^{+}\left(P_{n}^{+}\right) \neq$ $\emptyset$. So, we can obtain a finite or infinite impulsive point sequence $\left\{P_{n}\right\}$ in $M$ satisfying $P_{n}=F\left(P_{n-1}^{+}\right)=\Pi\left(P_{n-1}^{+}, T_{n-1}\right)$; here $P_{n-1}^{+}=I\left(P_{n}\right) \in N, T_{n-1} \in R_{+}$. Then we have

$$
\widetilde{\Pi}(P, t)= \begin{cases}\Pi(P, t), & 0 \leq t<T_{1}, \\ \Pi\left(P_{1}^{+}, t-T_{1}\right), & T_{1} \leq t<T_{1}+T_{2}, \\ \vdots & \\ \Pi\left(P_{n-1}^{+}, t-\sum_{k=1}^{n-1} T_{k}\right), & \sum_{k=1}^{n-1} T_{k} \leq t<\sum_{k=1}^{n} T_{k}, \\ \Pi\left(P_{N_{0}}^{+}, t-\sum_{k=1}^{N_{0}} T_{k}\right), & t \geq \sum_{k=1}^{N_{0}} T_{k}\end{cases}
$$

or

$$
\widetilde{\Pi}(P, t)= \begin{cases}\Pi(P, t), & 0 \leq t<T_{1}, \\ \Pi\left(P_{1}^{+}, t-T_{1}\right), & T_{1} \leq t<T_{1}+T_{2}, \\ \vdots & \\ \Pi\left(P_{n-1}^{+}, t-\sum_{k=1}^{n-1} T_{k}\right), & \sum_{k=1}^{n-1} T_{k} \leq t<\sum_{k=1}^{n} T_{k} . \\ \vdots & \end{cases}
$$

In system (2), $M=\left\{(x, y) \in R_{+} \mid x+y=h, x \geq 0, y \geq\right.$ $0\}, N=I(M)=\left\{(x, y) \in R_{+} \mid x=(1-\alpha) h, y=(1-\beta) h\right\}$. For the convenience, if $P \in X-M, F(P)$ is defined as the first point of intersection of $C^{+}(P)$ and $M$; that is, there exists a $t_{1} \in R_{+}$such that $F(P)=\Pi\left(P, t_{1}\right) \in M$, and for $0<t<$ $t_{1}, \Pi(P, t) \notin M$; if $B \in X-N, R(B)$ is defined as the first point of intersection of $C^{-}(P)$ and $N$; that is, there exists a $t_{2} \in R_{+}$such that $R(B)=\Pi\left(P,-t_{2}\right) \in N$, and for $-t_{2}<t<0$, $\Pi(B, t) \notin N$. Furthermore, the isocline $d x / d t=0$ is denoted by $L_{1}$; the isocline $d y / d t=0$ is denoted by $L_{2}$. The saddle separatrix $k_{1} x$ is denoted by $\Gamma$. Let $B$ be the intersection of the saddle separatrix $\Gamma$, and let $N$ and $C$ be the intersection of coordinate axis $x$ and set $N$. Next we will give the definition of the successor function of semidynamical system (2). First, we define a new number axis in set $N$. On straight line $y-$ $((1-\beta) x /(1-\alpha))=(1-\beta) h$ that corresponds to set $N$, take the origin at point $B$ and $\overrightarrow{B C}$ as positive direction and unit length to be consistent with coordinate axis $x$ or $y$; then we obtain a number axis $l$. For any $x \in l, l(x) \in N$.

Definition 4. A trajectory $\widetilde{\Pi}\left(P_{0}, t\right)$ is called order one periodic solution with period $T$ if there exists a point $P_{0} \in N$ and $T>0$ such that $P=F\left(P_{0}\right)=\Pi\left(P_{0}, T\right) \in M$ and $P^{+}=I(P)=P_{0}$.

Definition 5. Suppose $g: N \rightarrow N$ to be a map. For any $x \in$ $N$, there exists a $t_{1}>0$ such that $F(x)=\Pi\left(x, t_{1}\right)=x_{1} \in$ $M, x_{1}^{+}=I\left(x_{1}\right) \in N$; then $g(x)=l\left(x_{1}^{+}\right)-l(x)$ is called the successor function of point $x$, and the point $x_{1}^{+}$is called the successor point of $x$.

Remark 6. If $g\left(x_{0}\right)=0$, the trajectory $\Pi\left(x_{0}, t\right)$ with initial point $x_{0}$ is an order one periodic solution of system (2).
According to the continuity of compound function, we have the following.

\section{Lemma 7. The successor function $g(x)$ is continuous.}

In system (2), if there exist two points $x_{1} \in N, x_{2} \in$ $N$ satisfying $g\left(x_{1}\right) g\left(x_{2}\right)<0$, by the zero point theorem of continuous function in the closed interval, we know that there must be a point $x_{0} \in N$ which is between $x_{1}$ and $x_{2}$ such that $g\left(x_{0}\right)=0$, so we have the following lemmas.

Lemma 8 (existence theorem of order one periodic solution). If there exist two points $x_{1} \in N, x_{2} \in N$, which are both in the left $B$ or right $B$, satisfying $g\left(x_{1}\right) g\left(x_{2}\right)<0$, it has an order one periodic solution in system (2).

Lemma 9. Let $l_{1}=\{(x, y) \mid y=k x, x \geq 0, y \geq 0\}, l_{2}=$ $\{(x, y) \mid x+y=h, x \geq 0, y \geq 0\}=M, l_{3}=\{(x, y) \mid$ $y-((1-\beta) x /(1-\alpha))=(1-\beta) h, x \geq 0, y \geq 0\}=N$. Denote $F \in M$ as the intersect of $l_{1}$ and $l_{2}$ and $G \in N$ as the intersect of $l_{1}$ and $l_{3}$; then,
(i) if $\alpha>\beta, l\left(F^{+}\right)<l(G)$;
(ii) if $\alpha=\beta, l\left(F^{+}\right)=l(G)$;
(iii) if $\alpha<\beta, l\left(F^{+}\right)>l(G)$.

Proof. We focus on proving case (i), by similar method we can prove case (ii) and (iii). By computing, we can easily obtain $F(h /(k+1), k h /(k+1)), G((1-\alpha)(1-\beta) h /(k(1-\alpha)+(1-\beta))$, $k(1-\alpha)(1-\beta) h /(k(1-\alpha)+(1-\beta)))$, and $F^{+}(h(1-\alpha) /(k+1)$, $k(1-\beta) h /(k+1))$. If $\alpha>\beta$; then $(1-\alpha)(1-\beta) h /(k(1-\alpha)+$ $(1-\beta))>h(1-\alpha) /(k+1)$ and $k(1-\alpha)(1-\beta) h /(k(1-\alpha)+$ $(1-\beta))<k(1-\beta) h /(k+1)$. So, $l\left(F^{+}\right)<l(G)$. This completes the proof.

\section{Existence of Order One Periodic Solution of System (2)}

In this section, we will investigate the existence of order one periodic solution of system (2) by using the successor function.

We note that system (2) is an impulsive semidynamical system, where $M=\left\{(x, y) \mid x+y=h,(x, y) \in R_{2}^{+}\right\}$is the closed subset of $R_{2}^{+}$and $I:(x, y) \in M \rightarrow((1-\alpha) x$ and $(1-\beta) y) \in N$ is a continuous function. For any point $E$, let $x_{e}$ denote its abscissa, and let $y_{e}$ be its ordinate. If $E\left(x_{e}, y_{e}\right) \in$ $M$, then pulse occurs at point $E$, and the impulsive function transfers the point $E$ into $E^{+}$. Without loss of generality, unless otherwise specified we assume that the initial point of the trajectory lies in $N$.

Now we discuss the existence of order one periodic solution of system (2) from the following three cases.

Case $I$ ( $\alpha>\beta$ (see Figure 1)). In this case, according to Lemma $9, l\left(A^{+}\right)<l(B)$ and set $F\left(A^{+}\right)=A_{1}\left(x_{A_{1}}, y_{A_{1}}\right) \in M$; then impulse occurs at point $A_{1}$. Suppose point $A_{1}$ is subject to impulsive effects to point $A_{1}^{+}\left((1-\alpha) x_{A_{1}},(1-\beta) y_{A_{1}}\right) \in N$. In view of vector field and disjointness of any two trajectories, we know $x_{A}>x_{A_{1}}$ and $y_{A}<y_{A_{1}}$, so we have $x_{A^{+}}>x_{A_{1}^{+}}$and 


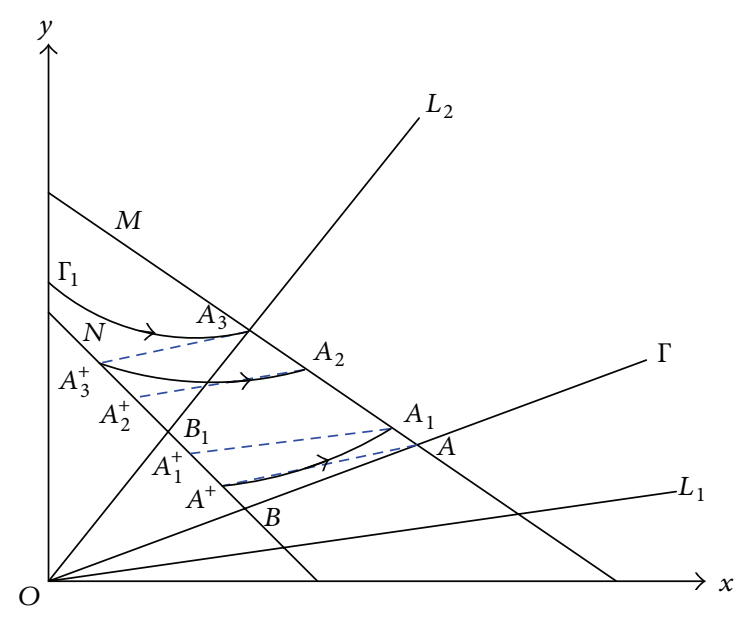

FIgURE 1

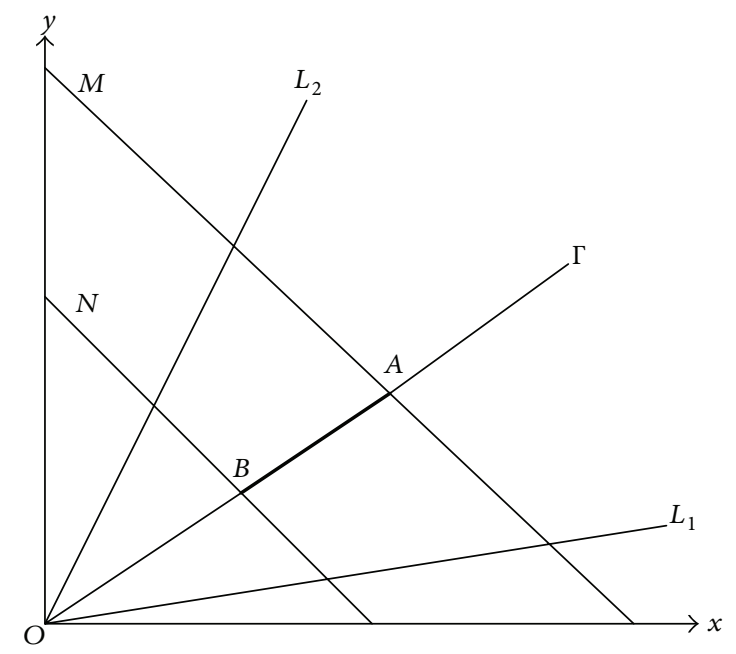

FIGURE 2

$y_{A^{+}}<y_{A_{1}^{+}}$; then $l\left(A_{1}^{+}\right)<l\left(A^{+}\right)$, and the successor function of $A^{+}$is $g\left(A^{+}\right)=l\left(A_{1}^{+}\right)-l\left(A^{+}\right)<0$; there exists a trajectory $\Gamma_{1}$ intersecting to $M$ at point $A_{3}$ which is subject to impulsive effects to point $A_{3}^{+} \in N$. Set $F\left(A_{3}^{+}\right)=A_{2} \in M$; then impulse occurs at point $A_{2}$. Suppose point $A_{2}$ is subject to impulsive effects to point $A_{2}^{+}\left((1-\alpha) x_{A_{2}},(1-\beta) y_{A_{2}}\right) \in N$. We know $x_{A_{2}}>x_{A_{3}}$ and $y_{A_{2}}<y_{A_{3}}$, so we have $x_{A_{2}^{+}}>x_{A_{3}^{+}}$and $y_{A_{2}^{+}}<$ $y_{A_{3}^{+}}$; then $l\left(A_{2}^{+}\right)>l\left(A_{3}^{+}\right)$, and the successor function of $A_{3}^{+}$is $g\left(A_{3}^{+}\right)=l\left(A_{2}^{+}\right)-l\left(A_{3}^{+}\right)>0$.

By Lemma 8 , we know there exists an order one periodic solution of system (2), whose initial point is between $A_{3}^{+}$and $A^{+}$in set $N$.

Case II ( $\alpha=\beta$ (see Figure 2)). In this case, according to Lemma 9, the point $A^{+}$coincides with $B$, and the successor function of $B$ is $g(B)=l(B)-l\left(A^{+}\right)=0$, so the segment $B A$ is an order one periodic solution of system (2).

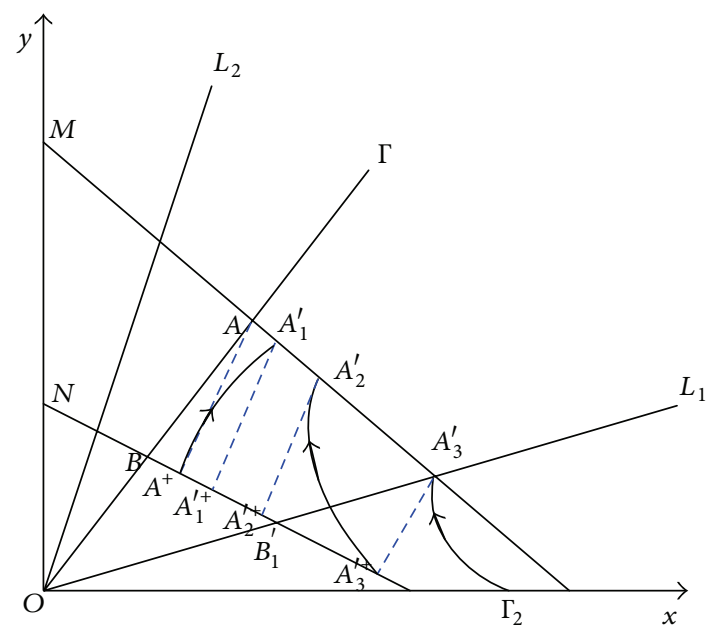

Figure 3

Case III $(\alpha<\beta$ (see Figure 3)). In this case, according to Lemma $9, l\left(A^{+}\right)>l(B)$ and set $F\left(A^{+}\right)=A_{1}^{\prime}\left(x_{A_{1}^{\prime}} y_{A_{1}^{\prime}}\right) \in M$; then impulse occurs at point $A_{1}^{\prime}$. Suppose point $A_{1}^{\prime}$ is subject to impulsive effects to point $A_{1}^{\prime+}\left((1-\alpha) x_{A_{1}^{\prime}},(1-\beta) y_{A_{1}^{\prime}}\right) \in N$. We know $x_{A}<x_{A_{1}^{\prime}}$ and $y_{A}>y_{A_{1}^{\prime}}$, so we have $x_{A^{+}}<x_{A_{1}^{\prime+}}$ and $y_{A^{+}}>y_{A_{1}^{\prime+}}$ then $l\left(A_{1}^{\prime+}\right)>l\left(A^{+}\right)$, and the successor function of $A^{+}$is $g\left(A^{+}\right)=l\left(A_{1}^{\prime+}\right)-l\left(A^{+}\right)>0$; there exists a trajectory $\Gamma_{1}$ intersecting to $M$ at point $A_{3}^{\prime}$ which is subject to impulsive effects to point $A_{3}^{\prime+} \in N$. Set $F\left(A_{3}^{\prime+}\right)=A_{2}^{\prime} \in M$; then impulse occurs at point $A_{2}^{\prime}$. Suppose point $A_{2}^{\prime}$ is subject to impulsive effects to point $A_{2}^{\prime}\left((1-\alpha) x_{A_{2}^{\prime}},(1-\beta) y_{A_{2}^{\prime}}\right) \epsilon$ $N$. We know $x_{A_{2}^{\prime}}<x_{A_{3}^{\prime}}$ and $y_{A_{2}^{\prime}}>y_{A_{3}^{\prime}}$, so we have $x_{A_{2}^{\prime+}}<x_{A_{3}^{\prime+}}$ and $y_{A_{2}^{\prime+}}>y_{A_{3}^{\prime+}}$; then $l\left(A_{2}^{\prime+}\right)<l\left(A_{3}^{\prime+}\right)$, and the successor function of $A_{3}^{\prime+}$ is $g\left(A_{3}^{\prime+}\right)=l\left(A_{2}^{\prime+}\right)-l\left(A_{3}^{\prime+}\right)<$ 0 .

By Lemma 8 we know there exists an order one periodic solution of system (2), whose initial point is between $A^{+}$and $A_{3}^{\prime+}$ in set $N$.

Thus we have the following theorem.

Theorem 10. If $a d-b c<0$, there exists an order one periodic solution in system (2).

\section{Attractiveness of Order One Periodic Solution of System (2)}

In this section, we discuss the attractiveness of system (2).

Theorem 11. Assume that conditions $\alpha>\beta$ and $a d-b c<0$ hold; then,

(a) there exist order one periodic solutions of system (2) in the region $A A_{3} A_{3}^{+} A^{+}$.

(b) If the periodic solution is unique, it is attractive, where the attractive region is in the region $A A_{3} A_{3}^{+} A^{+}$and 
the coordinate $A\left(h /\left(k_{1}+1\right), k_{1} h /\left(k_{1}+1\right)\right), A_{3}(d h /(c+$ d), $c h /(c+d)), A_{3}^{+}(d h(1-\alpha) /(c+d), \operatorname{ch}(1-\beta) /(c+d))$, $A^{+}(h(1-\alpha) /(k+1), k h(1-\beta) /(k+1))$ (see Figure 1$)$.

Proof. (a) By the derivation of Theorem 10 and the conditions $\alpha>\beta$, we know $g\left(A^{+}\right)=l\left(A_{1}^{+}\right)-l\left(A^{+}\right)<0, g\left(A_{3}^{+}\right)=l\left(A_{2}^{+}\right)-$ $l\left(A_{3}^{+}\right)>0$, so there exists an order one periodic solution at least of system (2) whose initial point is between $A_{3}^{+}$and $A^{+}$ in set $N$. According to the continuity of successor function and the zero point theorem of continuous function, we can easily prove there exists odd-numbered order one periodic solution of system (2).

(b) If the periodic solution is unique, assume trajectory $\widehat{P_{+} P}$ and segment $P P_{+}$are the periodic solution of system (2) with initial point $P_{+} \in N$ (see Figure 4). Next, we will prove the periodic solution is attractive.

On the one hand, set $F(C)=A_{1}$; then owning to the disjointness of any two trajectories, we have $x_{A_{1}}<x_{P}$ and $y_{A_{1}}>y_{P}$; thus, $x_{C}<x_{A_{1}^{+}}<x_{P^{+}}$and $y_{C}>y_{A_{1}^{+}}>y_{P^{+}}$. So, we obtain $l\left(A_{1}^{+}\right)>l(C)$ and $g(C)=l\left(A_{1}^{+}\right)-l(C)>0$; set $F\left(A_{1}^{+}\right)=$ $A_{2}$; we know $x_{A_{1}}<x_{A_{2}}<x_{P}$ and $y_{A_{1}}>y_{A_{2}}>y_{P}$; then we have $x_{A_{1}^{+}}<x_{A_{2}^{+}}<x_{P^{+}}$and $y_{A_{1}^{+}}>y_{A_{2}^{+}}>y_{P^{+}}$. So $l\left(A_{2}^{+}\right)>l\left(A_{1}^{+}\right)$ and $g\left(A_{1}^{+}\right)=l\left(A_{2}^{+}\right)-l\left(A_{1}^{+}\right)>0$. This process is continuing; then we get a sequence $\left\{A_{k}^{+}\right\}_{k=1,2, \ldots}$ of set $N$ satisfying $x_{A_{1}^{+}}<$ $x_{A_{2}^{+}}<\cdots<x_{A_{k}^{+}}<\cdots<x_{P^{+}}$and $y_{A_{1}^{+}}>y_{A_{2}^{+}}>\cdots>y_{A_{k}^{+}}>$ $\cdots>y_{P^{+}}$, so $l\left(A_{k+1}^{+}\right)>l\left(A_{k}^{+}\right)$, and $g\left(A_{k}^{+}\right)=l\left(A_{k+1}^{+}\right)-l\left(A_{k}^{+}\right)>$ 0 . Series $\left\{y_{A_{k}^{+}}\right\}_{k=1,2, \ldots}$ decreases monotonously and has lower bound, and series $\left\{x_{A_{k}^{+}}\right\}_{k=1,2, \ldots}$ increases monotonously and has upper bound, so $\lim _{k \rightarrow+\infty} x_{A_{k}^{+}}$and $\lim _{k \rightarrow+\infty} y_{A_{k}^{+}}$exist. Next we will prove $\lim _{k \rightarrow+\infty} x_{A_{k}^{+}}=x_{P^{+}}$and $\lim _{k \rightarrow+\infty} y_{A_{k}^{+}}=$ $y_{P^{+}}$. Set $A_{*}^{+}=\lim _{k \rightarrow+\infty} A_{k}^{+}$; that is, we will prove $A_{*}^{+}=P^{+}$. Otherwise, $A_{*}^{+} \neq P^{+}$, set $F\left(A_{*}^{+}\right)=\widetilde{A}$; then we have $x_{\widetilde{A}}<$ $x_{P}, x_{\widetilde{A}^{+}}<x_{P^{+}}$and $y_{\widetilde{A}}>y_{P}, y_{\widetilde{A}^{+}}>y_{P^{+}}$. Since $g\left(A_{*}^{+}\right) \geq 0$ and $A_{*}^{+} \neq P^{+}$, we have $g\left(A_{*}^{+}\right)=l\left(\widetilde{A}^{+}\right)-l\left(A_{*}^{+}\right)>0$; thus, we have $x_{A_{*}^{+}}<x_{\widetilde{A}^{+}}<x_{P}$ and $y_{A_{*}^{+}}>y_{\widetilde{A}^{+}}>y_{P}$ holds. Set $F\left(\widetilde{A}^{+}\right)=\stackrel{*}{\widetilde{A}}$; we know $x_{\widetilde{A}}<x_{\widetilde{\widetilde{A}}}<x_{P}, y_{\widetilde{A}}>y_{\widetilde{A}}>y_{P}$, and $x_{\widetilde{A}^{+}}<x_{\widetilde{A}^{+}}<x_{P^{+}}, y_{\widetilde{A}^{+}}>y_{\widetilde{A}^{+}}>y_{P^{+}}$; then we have $g\left(\widetilde{A}^{+}\right)=l\left(\widetilde{\widetilde{A}}^{+}\right)-l\left(\widetilde{A}^{+}\right)>0$, this is contradicted to the fact that $A_{*}^{+}$is a limit of sequence $\left\{A_{k}^{+}\right\}_{k=1,2, \ldots}$. So we obtain $A_{*}^{+}=P^{+}$ and $\lim _{k \rightarrow+\infty} x_{A_{k}^{+}}=x_{P^{+}}$and $\lim _{k \rightarrow+\infty} y_{A_{k}^{+}}=y_{P^{+}}$.

On the other hand, set $F(D)=B_{1}$; then owning to the disjointness of any two trajectories, we have $x_{B_{1}}>x_{P}$ and $y_{B_{1}}<y_{P}$; thus, $x_{D}>x_{B_{1}^{+}}>x_{P^{+}}$and $y_{D}<y_{B_{1}^{+}}<y_{P^{+}}$. So, $g(D)=l\left(B_{1}^{+}\right)-l(D)>0$; set $F\left(B_{1}^{+}\right)=B_{2}$. Because $x_{D}>x_{B_{1}^{+}}>x_{P^{+}}$and $y_{D}<y_{B_{1}^{+}}<y_{P^{+}}$, we know $x_{B_{1}}>x_{B_{2}}>$ $x_{P}$ and $y_{B_{1}}<y_{B_{2}}<y_{P}$; then we have $x_{B_{1}^{+}}>x_{B_{2}^{+}}>x_{P^{+}}$ and $y_{B_{1}^{+}}<y_{B_{2}^{+}}<y_{P^{+}}$. So $l\left(B_{2}^{+}\right)<l\left(B_{1}^{+}\right)$and $g\left(B_{1}^{+}\right)=$ $l\left(B_{2}^{+}\right)-l\left(B_{1}^{+}\right)<0$; this process is continuing; then we get a sequence $\left\{B_{k}^{+}\right\}_{k=1,2, \ldots}$ of set $N$ satisfying $x_{B_{1}^{+}}>x_{B_{2}^{+}}>\cdots>$ $x_{B_{k}^{+}}>\cdots>x_{P^{+}}$and $y_{B_{1}^{+}}<y_{B_{2}^{+}}<\cdots<y_{B_{k}^{+}}<\cdots<y_{P^{+}}$ so $l\left(B_{k+1}^{+}\right)<l\left(B_{k}^{+}\right)$and $g\left(B_{k}^{+}\right)=l\left(B_{k+1}^{+}\right)-l\left(B_{k}^{+}\right)<0$. Series $\left\{x_{B_{k}^{+}}\right\}_{k=1,2, \ldots}$ decreases monotonously and has lower bound and series $\left\{y_{B_{k}^{+}}\right\}_{k=1,2, \ldots}$ increases monotonously and has upper bound, so $\lim _{k \rightarrow+\infty} x_{B_{k}^{+}}$and $\lim _{k \rightarrow+\infty} y_{B_{k}^{+}}$exist. Next we will

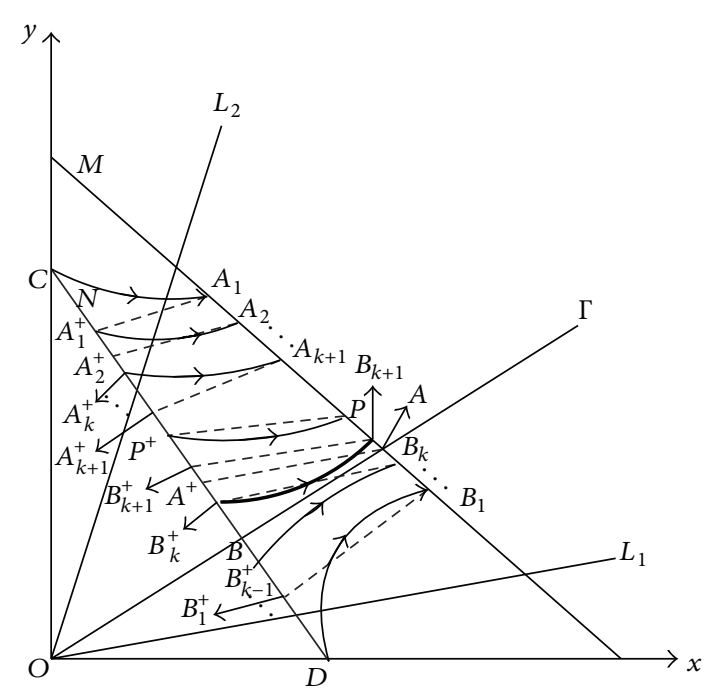

Figure 4

prove $\lim _{k \rightarrow+\infty} x_{B_{k}^{+}}=x_{P^{+}}$and $\lim _{k \rightarrow+\infty} y_{B_{k}^{+}}=y_{P^{+}}$. Set $B_{*}^{+}=\lim _{k \rightarrow+\infty} B_{k}^{+}$; that is, we will prove $B_{*}^{+}=P^{+}$. Otherwise, $B_{*}^{+} \neq P^{+}$, set $F\left(B_{*}^{+}\right)=\widetilde{B}$; then we have $x_{\widetilde{B}}>x_{P}, x_{\widetilde{B}^{+}}>x_{P^{+}}$, and $y_{\widetilde{B}}<y_{P}, y_{\widetilde{B}^{+}}<y_{P^{+}}$. Since $g\left(B_{*}^{+}\right) \geq 0$ and $B_{*}^{+} \leq P^{+}$; we have $g\left(B_{*}^{+}\right)=l\left(\widetilde{B}^{+}\right)-l\left(B_{*}^{+}\right)<0$; thus, we have $x_{B_{*}^{+}}>x_{\widetilde{B}^{+}}>x_{P}$, and $y_{B_{*}^{+}}<y_{\widetilde{B}^{+}}<y_{P}$ holds. Set $F\left(\widetilde{B}^{+}\right)=\widetilde{\widetilde{B}}$, we know $x_{\widetilde{B}}>x_{\widetilde{B}}>x_{P}$, $y_{\widetilde{B}}<y_{\widetilde{B}}<y_{P}$, and $x_{\widetilde{B}^{+}}>x_{\widetilde{B}^{+}}>x_{P^{+}}, y_{\widetilde{B}^{+}}<y_{\widetilde{B}^{+}}<y_{P^{+}}$; then we have $g\left(\widetilde{B}^{+}\right)=l\left(\widetilde{\widetilde{B}}^{+}\right)-l\left(\widetilde{B}^{+}\right)<0$, this is contradicted to the fact that $B_{*}^{+}$is a limit of sequence $\left\{B_{k}^{+}\right\}_{k=1,2, \ldots}$. So we obtain $B_{*}^{+}=P^{+}$and $\lim _{k \rightarrow+\infty} x_{B_{k}^{+}}=x_{P^{+}}$and $\lim _{k \rightarrow+\infty} y_{B_{k}^{+}}=y_{P^{+}}$. Since the trajectory initiating any point of $\Omega_{0}=\{(x, y) \mid x \geq$ $0, y \geq 0, x(0)+y(0)<h\}$ is certain to intersect with set $N$; next we only need to prove that the trajectory initiating any point of $N$ will ultimately tend to the unique order one periodic solution $\widehat{P^{+} P P^{+}}$.

Any point $U \in N$ must be in some interval $\left[l\left(B_{(k+1)}^{+}\right), l\left(B_{k}^{+}\right)\right)_{k=2,3, \ldots},\left[l\left(B_{1}^{+}\right), l(C)\right),\left(l(C), l\left(A_{1}^{+}\right)\right],\left(l\left(A_{k}^{+}\right)\right.$, $\left.l\left(A_{k+1}^{+}\right)\right]_{k=2,3, \ldots}$. Without loss of generality, we assume $U \in\left[l\left(B_{k+1}^{+}\right), l\left(B_{k}^{+}\right)\right)$. The trajectory initiating point $U$ moves between trajectory $\widehat{B_{k}^{+} B_{k+1}}$ and $\widehat{B_{k+1}^{+} B_{k+2}}$ and intersects with $M$ at some point between $B_{k+2}$ and $B_{k+1}$; under the impulsive effects it jumps to the point of $N$ which is between $\left[l\left(B_{k+1}^{+}\right), l\left(B_{k+2}^{+}\right)\right)$; then trajectory $\widetilde{\Pi}(U, t)$ continues to move between trajectory $\widehat{B_{k+1}^{+} B_{k+2}}$ and $\widehat{B_{k+2}^{+} B_{k+3}}$. This process can be continued unlimitedly. Since $\lim _{k \rightarrow+\infty} x_{B_{k}^{+}}=x_{P^{+}}$and $\lim _{k \rightarrow+\infty} y_{B_{k}^{+}}=y_{P^{+}}$, the intersection sequence of trajectory $\widetilde{\Pi}(U, t)$ and set $N$ will ultimately tend to point $P^{+}$. Thus the trajectory initiating any point of $N$ will ultimately tend to the unique order one periodic solution $\widehat{P^{+} P P^{+}}$.

From the above analysis, we know the trajectory initiating any point of $N$ will ultimately tend to order one periodic solution $\widehat{P^{+} P P^{+}}$. Therefore, order one periodic solution 


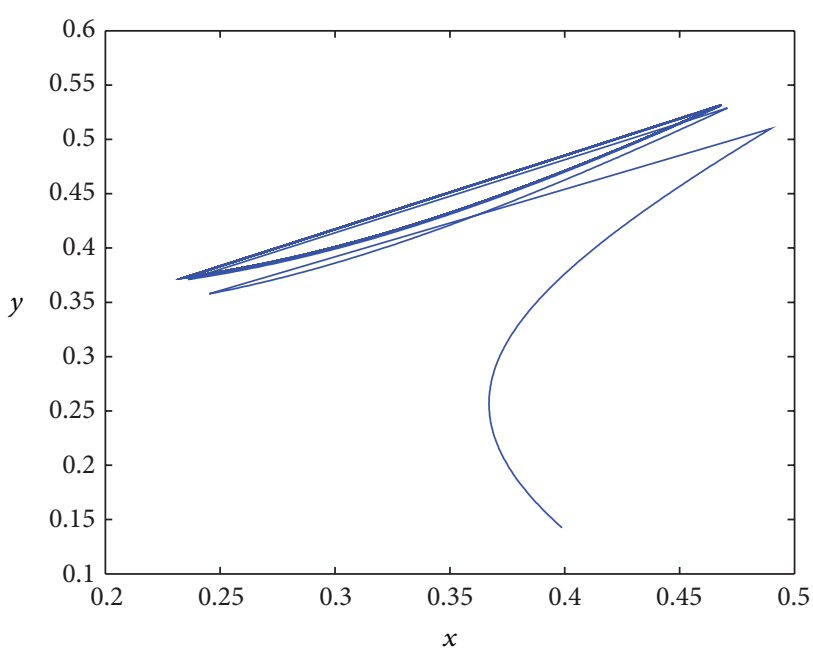

(a)

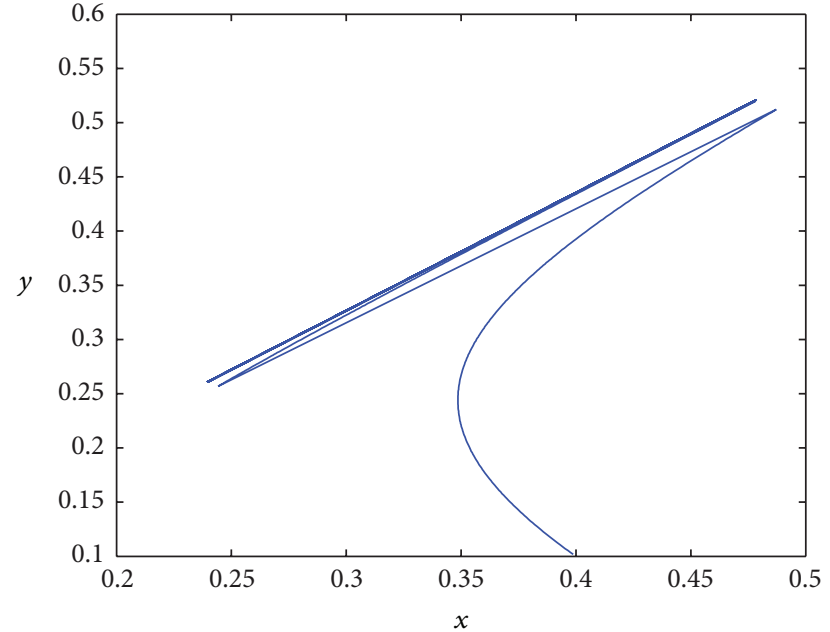

(b)

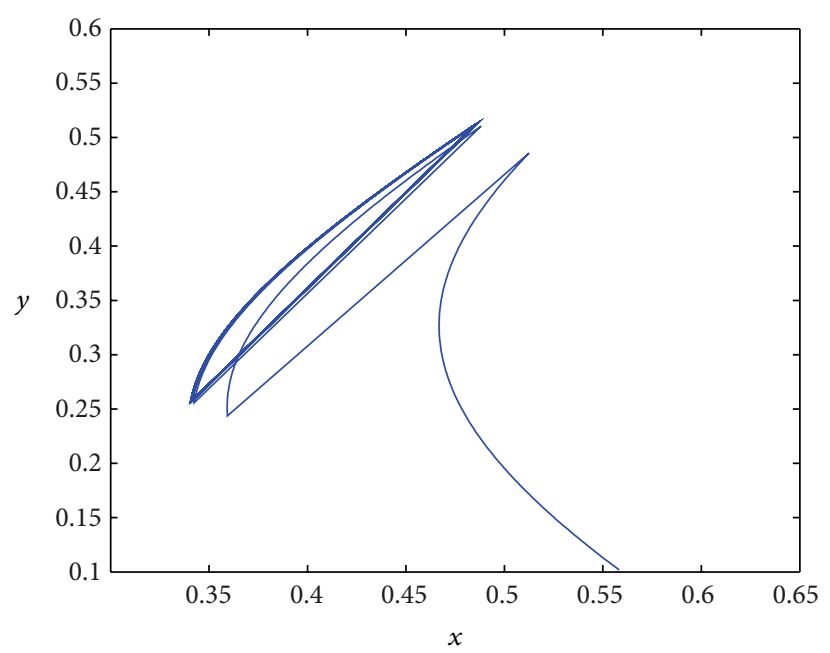

(c)

Figure 5: Phase portrait of system (2) to see the effect of the proportion of killed immature and mature $\alpha$ and $\beta$ with $a=0.6, b=0.8, c=0.5$, $d=0.2$, and $h=1$. (a) $\alpha>\beta$. (b) $\alpha=\beta$. (c) $\alpha<\beta$.

$\widehat{P^{+} P P^{+}}$is attractive in the region $\Omega_{0}=\{(x, y) \mid x \geq 0, y \geq$ $0, x(0)+y(0)<h\}$. This completes the proof. Like the analysis of Theorem 11, we have the following theorem.

Theorem 12. Assume that conditions $\alpha>\beta$ and $a d-b c<0$ hold; then,

(a) there exists an order one periodic solutions of system (2) in the region $A_{3}^{\prime} A A^{+} A_{3}^{\prime+}$.

(b) If the periodic solution is unique, it is attractive, where the attractive region is in the region $A_{3}^{\prime} A A^{+} A_{3}^{\prime+}$ and the coordinate $A_{3}^{\prime}(a h /(a+b), b h /(a+b)), A\left(h /\left(k_{1}+\right.\right.$ $\left.1), k_{1} h /\left(k_{1}+1\right)\right), \quad A^{+}(h(1-\alpha) /(k+1), k h(1-$ $\beta) /(k+1)) A_{3}^{\prime+}(a h(1-\alpha) /(a+b), b h(1-\beta) /(a+b))$ (see Figure 3).

Theorem 13. if $\alpha=\beta$ and $a d-b c<0$ hold, in view of vector field and Lemma 9, the segment $B A$ is a unique order one periodic solution of system (2) which is attractive in the region $\Omega_{0}=\{(x, y) \mid x \geq 0, y \geq 0, x(0)+y(0)<h\}$.

Proof. In view of vector field and Lemma 9, we can easily prove the theorem; thus, the proof is omitted.

\section{Conclusion}

To control pest, a stage-structure model with state-dependent impulsive control was investigated. We defined the successor functions of semicontinuous dynamic system and gave a new method to judge the existence of order one periodic solution of such system. By means of sequence convergence rules and qualitative analysis, we give sufficient conditions of the existence of order one periodic solution, as well as global attractiveness of order one periodic solution. To verify the theoretical results obtained in this paper, we will give some 


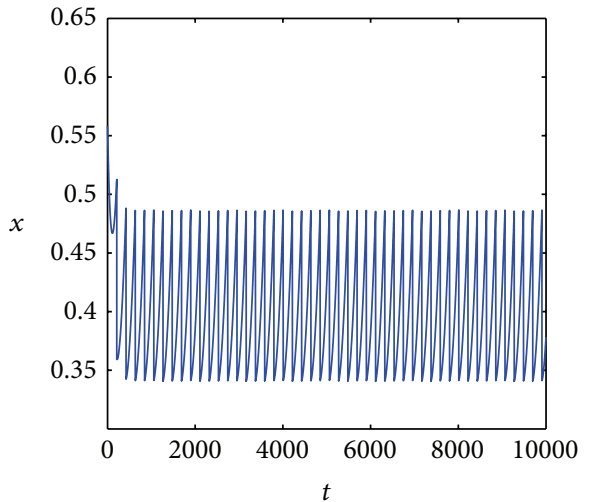

(a)

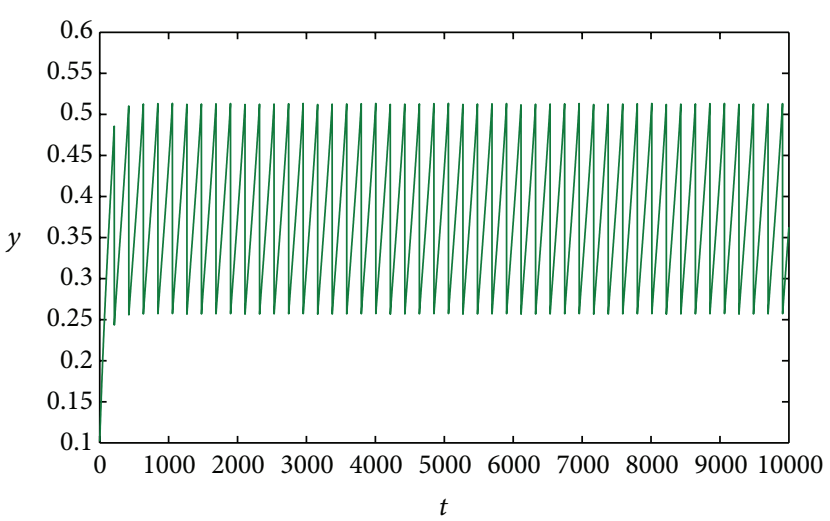

(b)

FIGURE 6: Permanence of system (2) to see the effect of the the proportion of killed immature and mature $\alpha$ and $\beta$. Other parameters are the same with those in Figure 5. (a) Time-series of the population $x(t)$. (b) Time-series of the population $y(t)$.

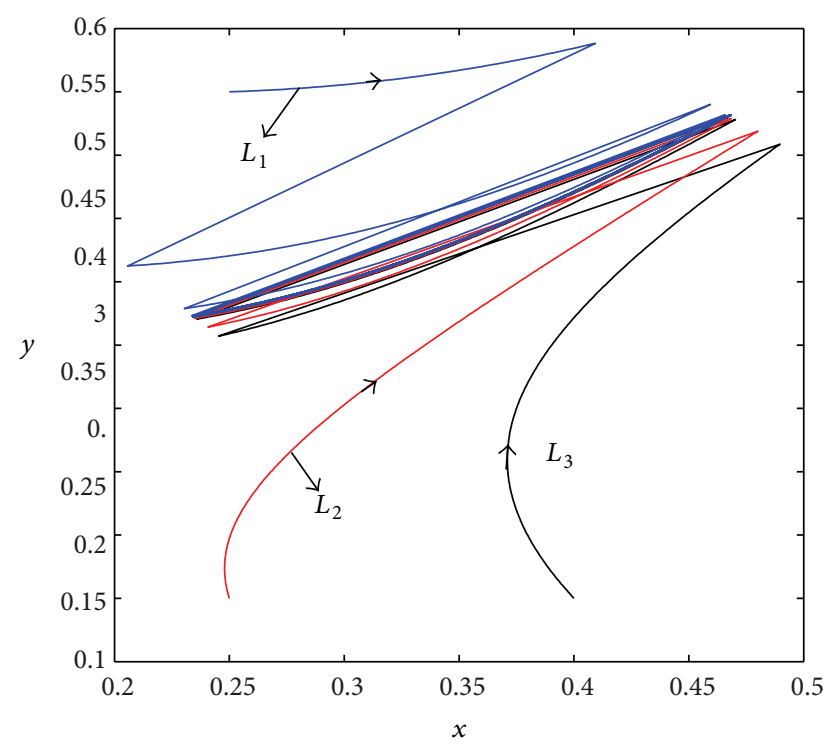

FIGURE 7: The global attractiveness of system (2) with parameters $a=0.6, b=0.8, c=0.5, d=0.2, h=0.2, \alpha=0.5, \beta=0.3$. The initial values for trajectory $L_{1}, L_{2}$ and $L_{3}$ are $(0.25,0.55),(0.25,0.15)$ and $(0.4,0.15)$, respectively.

numerical simulations. Let $a=0.6, b=0.8, c=0.5$, $d=0.2, h=1$. By comparing Figures 1,2 , and 3 with Figures $5(\mathrm{a}), 5(\mathrm{~b})$, and $5(\mathrm{c})$, it is obvious that our new method to judge the existence of order one periodic solution is precise. From Figures 6(a) and 6(b), using our spraying strategy, pest population can be controled under the economic threshold, whose method reduces insecticide application and protects environment. It is the first time to put forward the method. Our results have provided some fundamental theoretical conclusions that could be of applied importance.

Remark 14. The attractiveness of order one periodic solution of system (2) can be shown by Figure 7 . We take some different initial values, for example, $(0.25,0.55),(0.25,0.15)$ and $(0.4,0.15)$, respectively. By numerical simulations, we find that the trajectories initiating different values finally tend to a unique order one periodic solution of system (2). This indicates that the order one periodic solution of system (2) is globally attractive and we guess that it is unique order one periodic solution of system (2).

\section{Acknowledgments}

This work is supported by the National Natural Science Foundation of China (10971001) and the Excellent Talents Support Project of Universities and Colleges in Liaoning.

\section{References}

[1] R. M. Nisbet, S. P. Blythe, W. S. C. Gurney, and J. A. J. Metz, "Stage-structure models of populations with distinct growth and development processes," IMA Journal of Mathematics Applied, vol. 2, no. 1, pp. 57-68, 1985.

[2] R. M. Nisbet, W. S. C. Gurney, and J. A. J. Metz, "Stage structure models applied in evolutionary ecology," Biomathematics, vol. 18, pp. 428-449, 1989.

[3] S. A. Gourley and Y. Kuang, "A stage structured predator-prey model and its dependence on maturation delay and death rate," Journal of Mathematical Biology, vol. 49, no. 2, pp. 188-200, 2004.

[4] Y. Fan and L. Chen, "Periodic solutions and period-similarity of a growth model with age-structured population," Systems Science and Mathematical Sciences, vol. 4, no. 2, pp. 148-158, 1991.

[5] J. Cui, L. Chen, and W. Wang, "The effect of dispersal on population growth with stage-structure," Computers \& Mathematics with Applications, vol. 39, no. 1-2, pp. 91-102, 2000.

[6] X.-A. Zhang, L. Chen, and A. U. Neumann, "The stagestructured predator-prey model and optimal harvesting policy," Mathematical Biosciences, vol. 168, no. 2, pp. 201-210, 2000.

[7] Y. Xiao and L. Chen, "Analysis of a SIS epidemic model with stage structure and a delay," Communications in Nonlinear Science \& Numerical Simulation, vol. 6, no. 1, pp. 35-39, 2001. 
[8] Y. Xiao and L. Chen, "Effects of toxicants on a stage-structured population growth model," Applied Mathematics and Computation, vol. 123, no. 1, pp. 63-73, 2001.

[9] H. Zhang, L. Chen, and R. Zhu, "Permanence and extinction of a periodic predator-prey delay system with functional response and stage structure for prey," Applied Mathematics and Computation, vol. 184, no. 2, pp. 931-944, 2007.

[10] B. Liu, Q. Zhang, and Y. Gao, "The dynamics of pest control pollution model with age structure and time delay," Applied Mathematics and Computation, vol. 216, no. 10, pp. 2814-2823, 2010.

[11] S. Tang, J. Liang, Y. Xiao, and R. A. Cheke, "Sliding bifurcations of Filippov two stage pest control models with economic thresholds," SIAM Journal on Applied Mathematics, vol. 72, no. 4, pp. 1061-1080, 2012.

[12] S. Gao and L. Chen, "The effect of seasonal harvesting on a single-species discrete population model with stage structure and birth pulses," Chaos, Solitons \& Fractals, vol. 24, no. 4, pp. 1013-1023, 2005.

[13] V. Lakshmikantham, D. D. Bainnov, and P. S. Simeonov, Theory of Impulsive Differential Equations, vol. 6 of Series in Modern Applied Mathematics, World Scientific, Singapore, 1989.

[14] D. D. Bainov and P. S. Simeonov, Impulsive Differential Equations: Periodic Solutions and Applications, vol. 66 of Chapman and Hall/CRC Monographs and Surveys in Pure and Applied Mathematics, Longman Scientific \& Technical, New York, NY, USA, 1993.

[15] D. D. Baĭnov and P. S. Simeonov, Systems with Impulse Effect: Stability, Theory and Applications, John Wiley \& Sons, New York, NY, USA, 1989.

[16] Z. Jin, M. Haque, and Q. Liu, "Pulse vaccination in the periodic infection rate SIR epidemic model," International Journal of Biomathematics, vol. 1, no. 4, pp. 409-432, 2008.

[17] S. Sun and L. Chen, "Permanence and complexity of the ecoepidemiological model with impulsive perturbation," International Journal of Biomathematics, vol. 1, no. 2, pp. 121-132, 2008.

[18] Z. Xiong, Y. Xue, and S. Li, "A food chain system with holling IV functional responses and impulsive effect," International Journal of Biomathematics, vol. 1, no. 3, pp. 361-375, 2008.

[19] X. Meng and Z. Li, "The dynamics of plant disease models with continuous and impulsive cultural control strategies," Journal of Theoretical Biology, vol. 266, no. 1, pp. 29-40, 2010.

[20] S. Tang, G. Tang, and R. A. Cheke, "Optimum timing for integrated pest management: modelling rates of pesticide application and natural enemy releases," Journal of Theoretical Biology, vol. 264, no. 2, pp. 623-638, 2010.

[21] G. Jiang, Q. Lu, and L. Peng, "Impulsive control of a stagestructured pest management system," Journal of Mathematical Study, vol. 2, no. 2, pp. 329-344, 2005.

[22] S. Tang and R. A. Cheke, "State-dependent impulsive models of integrated pest management (IPM) strategies and their dynamic consequences," Journal of Mathematical Biology, vol. 50, no. 3, pp. 257-292, 2005.

[23] G. Jiang, Q. Lu, and L. Peng, "Impulsive ecological control of a stage-structured pest management system," Mathematical Biosciences and Engineering, vol. 2, no. 2, pp. 329-344, 2005. 


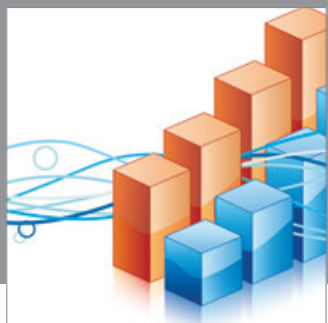

Advances in

Operations Research

mansans

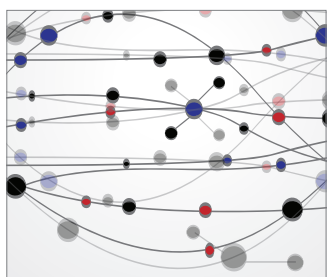

The Scientific World Journal
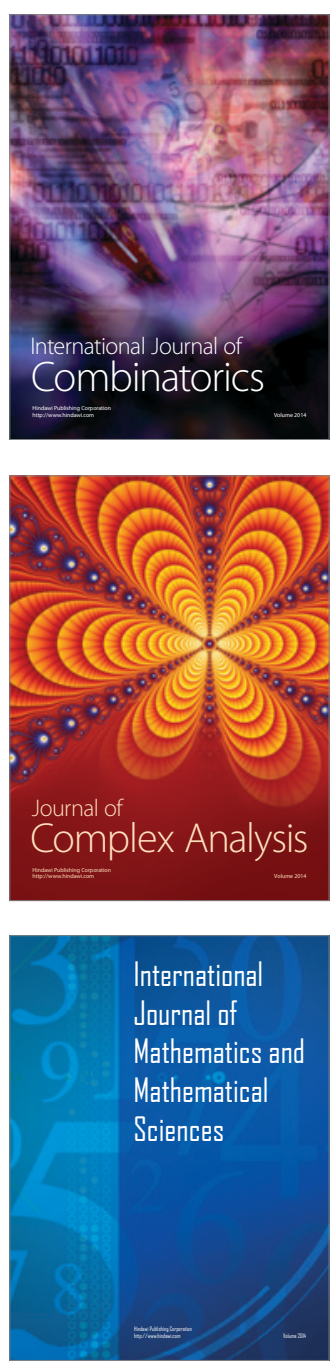
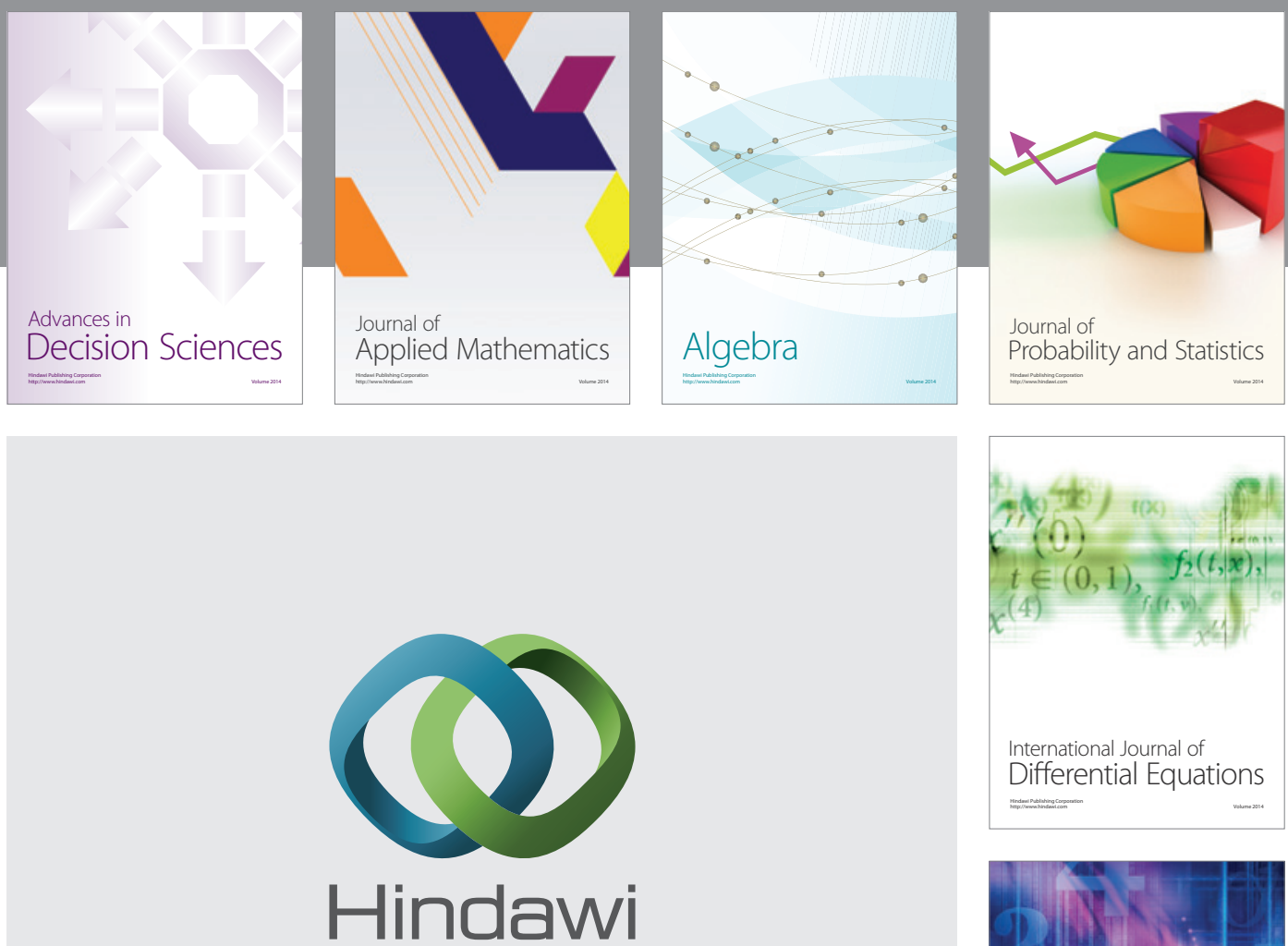

Submit your manuscripts at http://www.hindawi.com
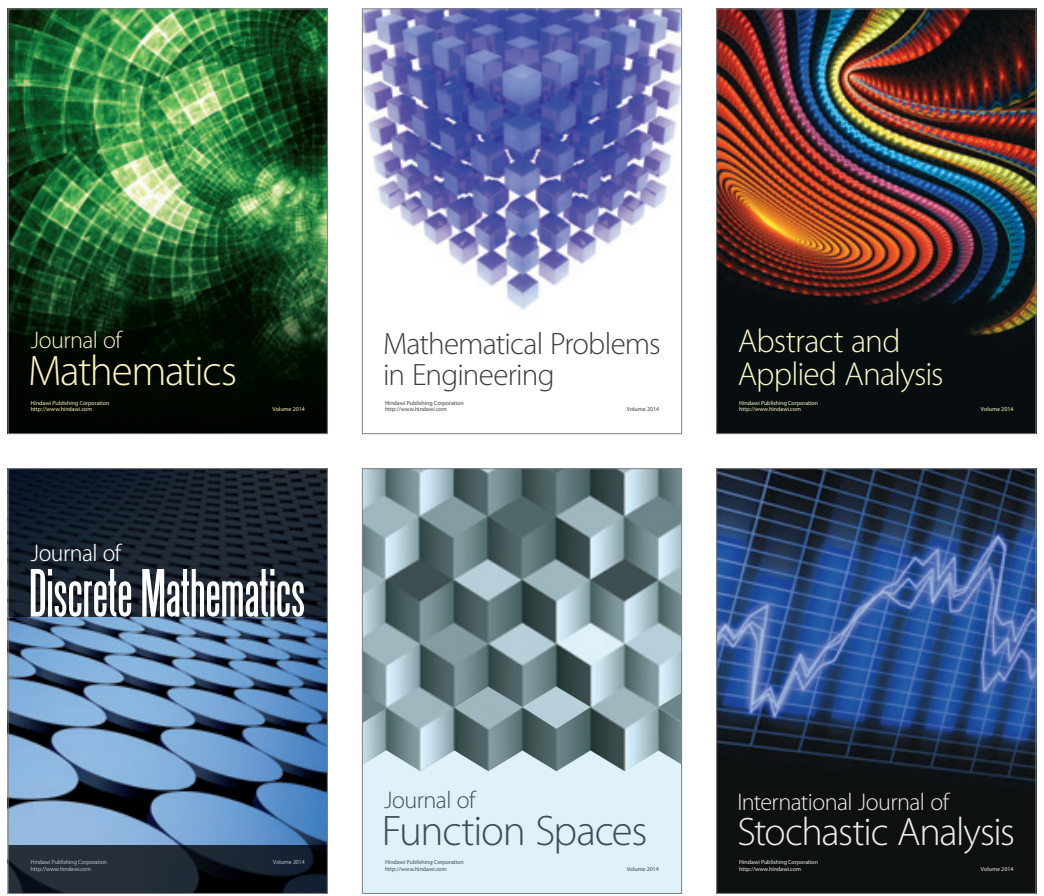

Journal of

Function Spaces

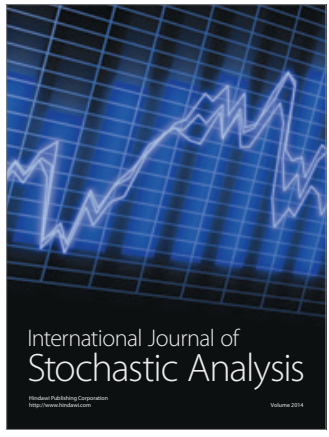

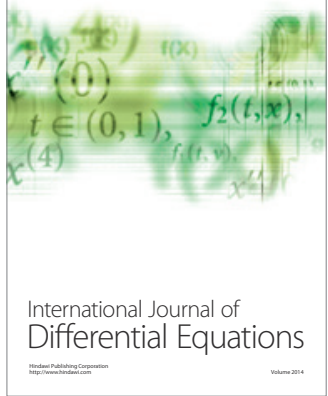
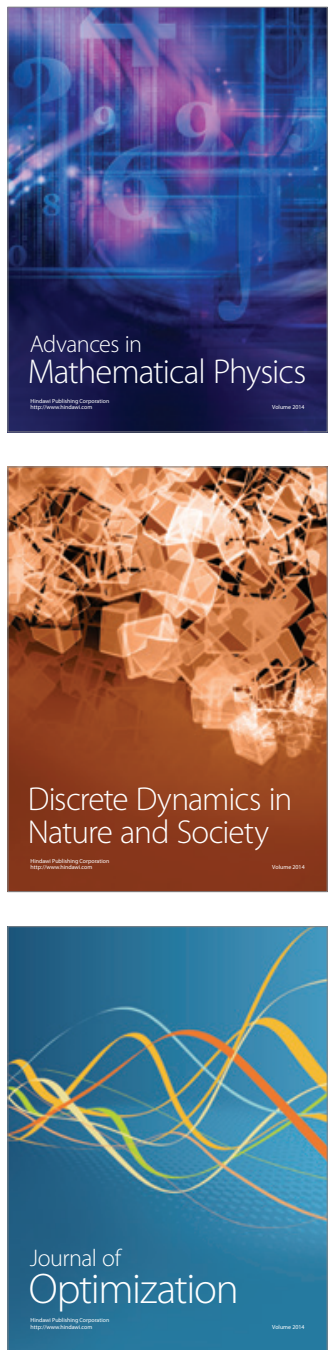